\title{
Reduced chromatin acetylation of malignant salivary gland tumors correlates with enhanced proliferation
}

\author{
Vivian Petersen Wagner ${ }^{1,2,3}$, Manoela Domingues Martins ${ }^{1,2,3}$, Douglas Magno Guimaraes ${ }^{3}$, \\ Artur Cunha Vasconcelos, ${ }^{1,2}$, Luise Meurer ${ }^{2,4}$, Pablo Agustin Vargas, Felipe Paiva Fonseca ${ }^{6}$, \\ Cristiane Helena Squarize ${ }^{3}$, Rogerio Moraes Castilho ${ }^{3}$ (iD \\ ${ }^{1}$ Department of Oral Pathology, School of Dentistry, Universidade Federal do Rio Grande do Sul, Porto Alegre, Rio Grande do Sul, \\ Brazil; ${ }^{2}$ Department of Experimental Pathology, Hospital de Clínicas de Porto Alegre, Porto Alegre, Rio Grande do Sul, Brazil; \\ ${ }^{3}$ Laboratory of Epithelial Biology, Department of Periodontics and Oral Medicine, University of Michigan School of Dentistry, Ann Arbor, \\ MI, USA; ${ }^{4}$ Department of Oral Medicine, Hospital de Clínicas de Porto Alegre (HCPA/UFRGS), Porto Alegre, Rio Grande do Sul, RS, \\ Brazil; ${ }^{5}$ Department of Oral Diagnosis, Piracicaba Dental School, University of Campinas, Piracicaba, São Paulo, Brazil; ${ }^{6}$ Department of \\ Clinics, Pathology and Surgery, Dental School, Federal University of Minas Gerais, Belo Horizonte, Brazil
}

BACKGROUND: Epigenetic changes refer to any heritable modification in gene expression independent of alterations in the DNA sequence. Currently, it is well established that epigenetics represents a crucial player for tumor development. Nevertheless, the epigenetic mechanisms involved in the development and progression of salivary gland tumors (SGTs) remain poorly understood. METHODS: In this study, we analyzed the pattern of acetyl-histone $\mathrm{H} 3$ (lys9) expression in benign and malignant SGTs and further correlate our results with tumors' proliferative activity and clinical outcomes. We assembled tissue microarrays (TMAs) of 84 cases of SGTs and analyzed for acetyl-histone $\mathrm{H3}$ (lys9) and Ki-67 using immunohistochemistry. The study comprised 42 benign and 42 malignant SGTs.

RESULTS: All cases included in this study were positive to acetyl-H3 (lys9). We observed that malignant SGTs were hypoacetylated compared with benign $(P=0.04)$. Moreover, acetyl-H3 (lys9) expression was inversely correlated with Ki67 (**P=0.02).

CONCLUSION: This study provides the first insight regarding histone modifications in SGTs. Our results suggest that epigenetic mechanism, particularly hypoacetylation of histone $\mathrm{H} 3$ (lys9), might play a role in the behavior of salivary gland tumors. Also, our findings suggest that interfering with the acetylation pattern of tumor histones represents a potential novel therapeutic strategy for the treatment of SGTs.

J Oral Pathol Med (2017) 46: 792-797

Correspondence: Rogerio Moraes Castilho, Laboratory of Epithelial Biology, Department of Periodontics and Oral Medicine, University of Michigan, 1011 N University Ave, Room 2029C, Ann Arbor, MI, 481091078, USA. Tel: (734) 615-3406, Fax: (734) 763-5503,

E-mail: rcastilh@umich.edu

Accepted for publication January 30, 2017
Keywords: acetylation; epigenetics; head and neck cancer; histone; immunohistochemistry

\section{Introduction}

Salivary gland tumors (SGTs) represent a heterogeneous group of lesions that account for 5\% of all head and neck neoplasms, with an incidence of $0.4-13.5$ cases per 100.000 inhabitants annually (1). The management of malignant SGTs still represents a major challenge in head and neck oncology due to their biological heterogeneity that leads to an unpredictable response to therapy. No improvement was achieved in the overall survival of malignant SGTs over the last decades. The 5-years survival rate is $60-80 \%$, (2) although the histological subgrade presents a major impact in response to therapy leading to 5-years survival rates that contrast from over $90 \%$ in low-grade adenocarcinomas to as low as $37 \%$ in high-grade tumors $(1,3)$. Moreover, the low overall survival rates observed in long-term periods demonstrate that more efforts need to be addressed in this field. It is of paramount importance to expand our knowledge regarding the basic biological and molecular features of SGTs to identify more efficient therapeutic strategies further. In this context, epigenetics emerges as a promising field to better understand the behavior of SGTs, and as a promising target for therapy, using new developed Epi-drugs. Epigenetic changes refer to any heritable modification in gene expression independent of alterations in the DNA sequence. Epigenetic regulation of gene expression is mediated by DNA methylation, RNA-mediated silencing, and histone modifications, including lysine acetylation, methylation, and ubiquitination, serine phosphorylation, and arginine methylation (4). The dynamic changes in chromatin architecture, which might lead to modification of the transcriptional activity, are mainly driven by histone acetylation and deacetylation regulated by the activity of histone acetyltransferase (HAT) and histone 
deacetylase (HDAC). In histone acetylation, acetyl groups are added by HAT to the lysine residue resulting in neutralization of the positive charge in the histone tail leading to a reduced histone-DNA interaction. This process results in open or active chromatin structure while the opposite process, of histone deacetylation, results in chromatin condensation through the removal of acetyl groups by HDAC (4).

Previous studies established a significant correlation between histone modifications and tumor development. Histone deacetylation by HDACs impacts the expression of several genes involved in tumor initiation and progression (reviewed in (5)). Indeed, acetylation of histones is commonly lost in malignant cells when compared to their normal counterparts (6). The role of histone acetylation and HDACs, including its prognostic and therapeutic potential, has been increasingly explored in different types of cancer, including tumors of glandular origin. It has been demonstrated that increased expression of HDAC-1 is associated with higher proliferative capacity in pancreatic adenocarcinomas patients (7). Moreover, HDAC inhibitors decrease cell growth and increase apoptosis of pancreatic adenocarcinomas cell lines (8). In breast cancer, the use of HDAC inhibitors has already been tested in several phase I and II clinical trials, which revealed encouraging results demonstrating promising activity in reversing hormone resistance associated with little adverse effects (9).

In SGTs, other epigenetic events, such as DNA methylation, have been well explored. Most studies focused on adenoid cystic carcinoma (AdCC) and demonstrated increased methylation in several tumor suppressor genes (10). However, the pattern of histone acetylation, in AdCC as in the other SGTs, remains unexplored. Our group has deeply investigated the biological and clinical significance of histone 3 (H3) acetylation at Lys9 (11-13), (L.P. Webber, V.P. Wagner, M. Curra, P.A. Vargas, L. Meurer, V.C. Carrard, C.H. Squarize, R.M. Castilho \& M.D. Martins, unpublished data). Functional acetylation of H3 at Lys 9 is mainly associated with histone deposition, chromatin assembly, and gene activation (14). Our group demonstrated that head and neck squamous cell carcinoma cells are hypoacetylated (12) and this profile is associated with patients' poor prognosis (L.P. Webber, V.P. Wagner, M. Curra, P.A. Vargas, L. Meurer, V.C. Carrard, C.H. Squarize, R.M. Castilho \& M.D. Martins, unpublished data). We also observed that inhibition of HDAC reduces cisplatin resistance along with a decrease in the number of cancer stem cells (CSC) of head and neck squamous cell carcinoma (12, 13) and mucoepidermoid carcinoma (11).

The immunohistochemical expression of H3 (lys9) acetylation has not been investigated in SGTs. Therefore, the aim of this study was to evaluate the expression pattern of acetyl-H3 (lys9) in a panel comprising the most common benign and malignant SGTs. Further, we explored the correlation of acetyl-H3 (lys9) with lesions' proliferative potential through Ki67 immunostaining.

\section{Methods}

\section{Study population}

Eighty-four formalin-fixed, paraffin-embedded tissue blocks of SGTs were retrieved from the archives of two Brazilian pathology services (Piracicaba Dental School-University of Campinas and Porto Alegre University Hospital-Federal University of Rio Grande do Sul). Three oral pathologists reviewed the original hematoxylin-eosin-stained slides and confirmed the diagnoses according to the World Health Organization's 2005 Histological Typing of Salivary Gland Tumors classification. Clinical data including age, gender, and tumor localization were retrieved from medical files. The follow-up period was defined as the time from diagnosis until the last visit to the hospital or date of death. This study was approved by the Human Research Ethics Committee at the Federal University of Rio Grande do Sul (protocol number 130152/12).

\section{Tissue microarray construction}

Tissue microarray (TMA) construction was performed as previously described and validated $(15,16)$ by two trained oral pathologists. Briefly, tumor areas from the central and most cellular zone of the lesion were selected and marked on hematoxylin-eosin-stained sections using an objective marker (Nikon Corp, Tokyo, Japan). The slide was overlaid on the original paraffin block to determine the matching area to be used. A manual tissue arrayer (Sakura Co, Japan) was used, and three representative cylindrical cores of $2.0 \mathrm{~mm}$ in diameter were taken from each tissue block and arranged sequentially in a ready-to-use recipient paraffin block (Sakura Co, Japan). Two cores of the normal salivary gland and one core of oral squamous cell carcinoma were inserted in the left upper corner of each recipient block for orientation. A map specifying the precise location of each case was prepared to allow interpretation of the immunohistochemical results.

\section{Immunohistochemistry}

For immunohistochemical staining, TMA samples were sectioned into $3-\mu \mathrm{m}$ sections and placed on silanized slides. Sections were dewaxed in xylene solution and rehydrated in a descending ethanol series. The avidin-biotin blocking kit was used to block non-specific binding (Kit Vector Laboratories, Burlingame, CA, USA). Slides were incubated overnight with primary antibodies acetyl-H3 (lys9) (C5B11, 1:200; Cell Signaling, Danvers, MA, USA) and Ki67 (MIB1, 1:50; Dako, Glostrup, Denmark). Positive reactions were detected using the chromogen substrate diaminobenzidine tetrahydrochloride (DAB, Sigma-Aldrich Corp., St. Louis, MO, USA). Slides were counterstained with Mayer's hematoxylin. Sections of oral squamous cell carcinoma were used as positive controls, and negative controls were achieved by omitting the primary antibody.

Two pathologists using a double-headed microscope scored the histone H3 (lys9)-immunostained sections. Nuclear expression of tumor cells was categorized semiquantitatively by percentage of positive cells in a $200 \times$ magnification field, as follows: $1=0-50 \%$ of positive cells, $2=51-100 \%$ of positive cells.

Proliferative labeling index (PLI) was determined using $\mathrm{Ki}-67$-immunostained sections. Images of the selected fields were captured using a conventional light microscope (CX41RF model; Olympus Latin America, Inc., Miami, FL, USA) coupled to a color camera (QColor 5, Coolet, RTV; Olympus Inc.) and connected to a computer (Dimension 5150; Dell, Porto Alegre, RS, Brazil). The images were 
analyzed using version 2.18 QCapture software program (Quantitative Imaging Corporation, Inc., Surrey, DC, Canada). The total number and percentage of cells presenting nuclear positivity were assessed by counting 500 cells at a magnification of $400 \times$ in each core $(3$ cores $=1500$ cells per case). The results were expressed as the percentage of positive cells (mean and standard deviation).

\section{Statistical analysis}

All clinical and immunohistochemical data were analyzed with version 18.0 SPSS for Windows. Chi-square test, Fisher's exact test, or Student's $t$-test was used to compare clinicopathological features between benign and malignant SGTs. Kruskal-Wallis test, Mann-Whitney $U$-test, chisquare test, or Fisher's exact test was used to compare the immunohistochemical profile of $\mathrm{Ki} 67$ and $\mathrm{H} 3$ between benign and malignant SGTs. Spearman's correlation coefficient was used to examine the relationship between $\mathrm{H} 3$ and Ki67 expression. For all tests, statistical significance was defined as probability value $<0.05$.

\section{Results}

Clinicopathological aspects differ between benign and malignant SGTs

Forty-two cases of benign SGTs and 42 malignant SGTs were included in this study. The cases consisted of 33 pleomorphic adenomas (PA), nine Warthin's tumor (WT), 22 adenoid cystic carcinomas (AdCC), 15 mucoepidermoid carcinomas (MEC), and five acinic cell carcinoma (AcCC). Analyzing the entire cohort of patients, we observed a mean age at the time of diagnosis of $50.82( \pm 18.08)$ and a male: female ratio of 1:1.10. Major salivary glands were affected in $71.8 \%$ of cases $(82.14 \%$ in the parotid gland and $17.86 \%$ in the submandibular gland). Minor salivary glands were affected in $28.2 \%$ of cases (39.13\% in the palate). Table 1 compares the primary clinical data between benign and malignant SGTs. Benign SGTs were diagnosed in older patients $(P=0.03$ - Student's $t$-test $)$ and were located mainly in major salivary glands $(P=0.001-$ Fisher's exact test) when compared to malignant SGTs.

Benign SGTs are hyperacetylated compared with malignant SGTs

All cases included in this study were positive to acetyl-H3 (lys9). A significant difference was observed between

Table 1 Clinicopathological aspects in benign and malignant SGTs

\begin{tabular}{lccc}
\hline & Benign & Malignant & P value \\
\hline Gender (\%) & $21(50)$ & $18(45)$ & \\
$\quad$ Male & $21(50)$ & $22(55)$ & $0.66^{\mathrm{a}}$ \\
Female & $54.9( \pm 15.94)$ & $46.55( \pm 19.37)$ & $\mathbf{0 . 0 3}^{\mathrm{b}}$ \\
Age & & & \\
Site $(\%)$ & $56(87.8)$ & $20(54.1)$ & \\
$\quad$ Major & $5(12.8)$ & $17(45.9)$ & $\mathbf{0 . 0 0 1}^{\mathrm{a}}$ \\
Minor & &
\end{tabular}

Bold values denote significant difference among benign and malignant SGTs. ${ }^{a}$ Fisher's exact test.

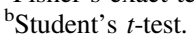

benign and malignant SGTs $(P=0.04-$ Fisher's exact test $)$ (Fig. 1) (Table 2). Benign SGT showed a higher percentage of cases presenting acetyl-H3 (lys9) expression in more than $50 \%$ of neoplastic cells (Fig. 1-arrows indicate positive tumor cells), thus suggesting that these lesions were hyperacetylated compared with malignant SGT. In PA, the immunopositivity was observed in the majority of both epithelial luminal duct cells and myoepithelial modified cells. In WT, despite finding a strong positivity in lymphoid cells, only epithelial cells were evaluated. We found a strong positivity in the epithelial cells of WT (Fig. 1A). In malignant SGTs, however, we noted a decrease in acetyl-H3 (lys9)-positive cells (Fig. 1A-arrowhead indicates negative tumor cells). We observed that the expression of acetylH3 (lys9) was equally distributed between epithelial and myoepithelial cells of AdCC, and among epidermoid, intermediate, and mucous cells of MEC (Fig. 1A). Also, Among all malignant tumors analyzed, both MEC and AcCC were the most hypoacetylated compared to AdCC. However, no significant difference was observed $(P=0.15$, chi-square test). Statistical analysis revealed a higher percentage of benign SGT presenting acetyl-H3 (lys9) expression in more than $50 \%$ of neoplastic cells $(P=0.04$ -Fisher's exact test) (Fig. 1B).

\section{Acetyl-H3 (lys9) is inversely correlated with proliferative} activity

In this study, benign SGTs showed a smaller PLI compared with malignant SGTs (Fig. 2A,B mean \pm SD of $0.67 \pm 0.81)$. We observed that some benign cases were strictly negative to Ki67. Thus, the PLI ranged from 0 to 2.6 (Fig. 2B). On the other hand, in malignant SGTs, the PLI ranged from 0.4 to 29.4 , with a significantly higher mean \pm SD of $8.59 \pm 8.2$ when compared to benign tumors $(P<0.001$, Mann-Whitney $U$-test $)$ (Fig. 2B). Among malignant SGTs, MEC had the highest PLI of $16.60 \pm 10.04$; however, no significant difference was observed with AdCC or AcCC $(P>0.05$, Kruskal-Wallis) (Fig. 2B). We also found a significant inverse correlation between acetyl-H3 (lys9) and Ki67 expression in SGT $(r=-0.34 ; P=0.02)$ (Fig. 2C). Therefore, hypoacetylation, represented by the decrease in acetyl-H3 (lys9) expression, was correlated with increased proliferative activity.

Histone H3 (lys9) acetylation association with outcome Information regarding clinical staging and outcome was available for 22 malignant SGTs. Among those cases, 10 $(45.5 \%)$ were classified at clinical stage I/II at the time of diagnosis and $22(54.5 \%)$ at stage III/IV. In advanced cases (III/IV), $66.7 \%$ of patients presented hypoacetylated H3 (Lys9) lesions, compared with $50 \%$ in the early cases. Despite the slight tendency observed, no significant difference was observed ( $P=0.36$, Fisher's exact test). During the follow-up period (between 4 and 13 years), three patients deceased, of which $2(66.7 \%)$ presented hypoacetylated lesions.

\section{Discussion}

SGTs are an extremely heterogeneous group of neoplasms that raise important awareness for its unpredictable clinical 


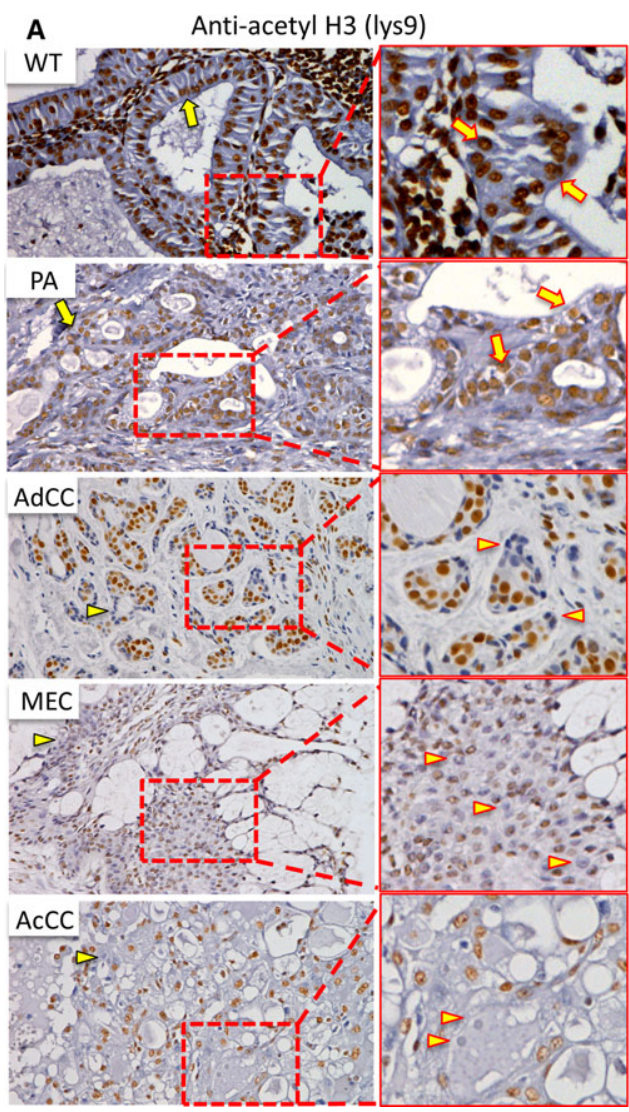

B

Figure 1 Immunohistochemical expression of acetyl-histone H3 (lys9) in salivary gland tumors. (A) Two benign salivary gland tumors (pleomorphic adenoma - PA, Warthin's tumor-WT) and three malignant salivary gland tumors (adenoid cystic carcinoma-AdCC, mucoepidermoid carcinoma—MEC, and acinic cell carcinoma-AcCC) are depicted. Note a high number of positive nuclear staining in benign salivary gland tumors (arrow) compared with its malignant counterpart (arrowhead). (B) Diagram represents benign and malignant tumor samples expressing more than 50\% of tumor cells expressing acetylH3 (lys9) (black) or less that $50 \%$ of tumor cells expressing acetyl-H3 (lys9) (gray).

Table 2 Expression of acetyl-H3 (Lys9) in SGTs

\begin{tabular}{lccccc}
\hline ac. H3(lys9) & $P A(\%)$ & $W T(\%)$ & $A d C C(\%)$ & $M E C(\%)$ & $A c C C(\%)$ \\
\hline$<50 \%$ & $11(33.3)^{\mathrm{a}}$ & $2(22.2)^{\mathrm{a}}$ & $14(63.6)^{\mathrm{b}}$ & $6(40)^{\mathrm{a}, \mathrm{b}}$ & $2(40)^{\mathrm{a}, \mathrm{b}}$ \\
$>50 \%$ & $28(66.7)$ & $7(77.8)$ & $8(36.4)$ & $9(60)$ & $3(60)$ \\
\hline
\end{tabular}

Absolute number and percentage of cases according to ac. H3(lys9) expression.

Different letters denote significant difference among column proportions $(P<0.05-z$-test $)$.

outcomes (1). Other peculiar characteristics of SGTs, such as unknown etiological factors (17), increased incidence in pediatric patients (18), and low survival rates in the longterm analysis (2), support the concern of health professionals. The recent discoveries of specific genetic alterations have deepened our knowledge about the molecular biology SGTs (19); nevertheless, targeted therapy still presents a very limited role in the management of malignant SGTs. While enough efforts are addressed in the field of genetics, epigenetics of SGTs remains somewhat obscure. In the present study, we analyzed for the first time the histone acetylation profile of most common benign and malignant SGTs. Our results revealed that malignant tumors are hypoacetylated and therefore present a more condensed chromatin compared with benign tumors (Fig. 3).
Moreover, acetylation of H3 (lys9) was inversely correlated with the proliferative activity of SGTs.

The etiology of cancer was for long associated essentially with genetic aberrations. However, over the past decades, epigenetics emerged as a crucial player through tumor development, allowing the understanding of gene expression modulation in the absence of DNA sequence alterations. Among epigenetic mechanisms, histone modifications present a primary role in chromatin stability and packing, thus controlling gene expression and silencing. Chromatin decondensation is a key mechanism that guarantees gene transcription and repair of the genome, regulated mainly by histone acetylation (4). In the present study, we observed that most common benign SGTs, PA and WT, were hyperacetylated compared with malignant SGT. Global histone acetylation is involved in the process of cellular differentiation and is often seen downregulated in pluripotent ES cells. As ES cells undergo differentiation, the acetylation of histones, more specifically histone $\mathrm{H} 3$ at lysine 9 , is observed (20). Indeed, we found that more differentiated cells, present in benign tumors, present enhanced acetylation of histones.

On the other hand, malignant tumors showed fewer acetylhistone H3 (lys9) expression. Chromatin organization plays an important role during DNA repair. Histone acetylation opens chromatin, allowing repair proteins to gain access to the 
A

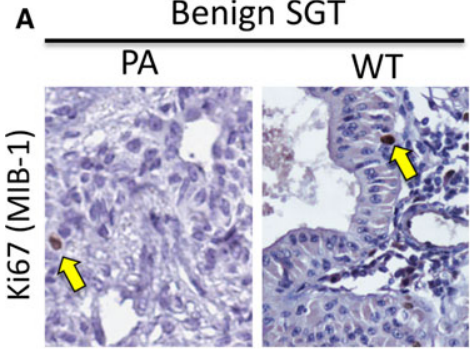

B

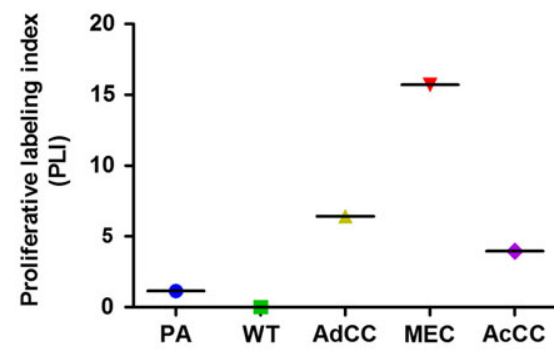

Malignant SGT

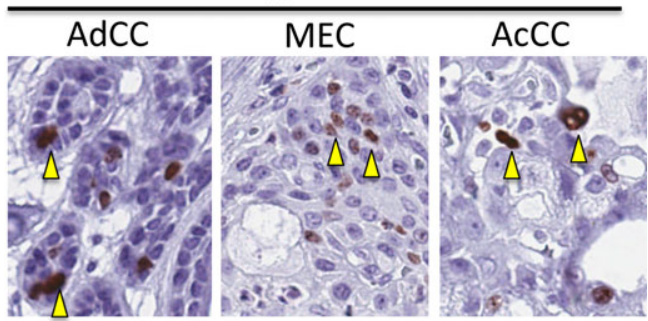

C

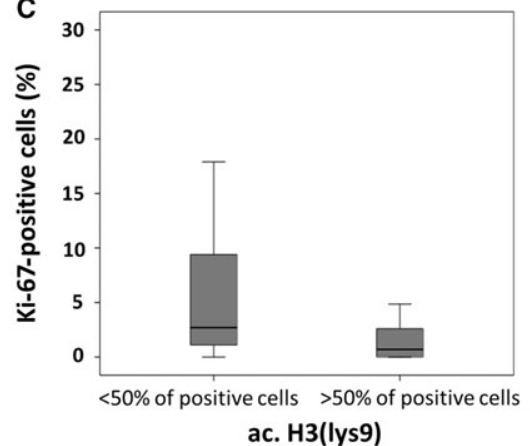

Figure 2 Correlation between cellular proliferation and histone acetylation in salivary gland tumors. (A) Immunohistochemical staining of Ki-67 in salivary gland tumors. Note little amount of positive cells present in benign salivary gland tumors (arrow) compared with malignant salivary gland tumors (arrowhead). (B) Proliferative labeling index (PLI) of each of the salivary gland tumors accordingly to the quantification of Ki-67-positive cells. (C) Expression of Ki-67 in tumors cells and its correlation with histone acetylation levels (ac. H3 (lys9) in salivary gland tumors.

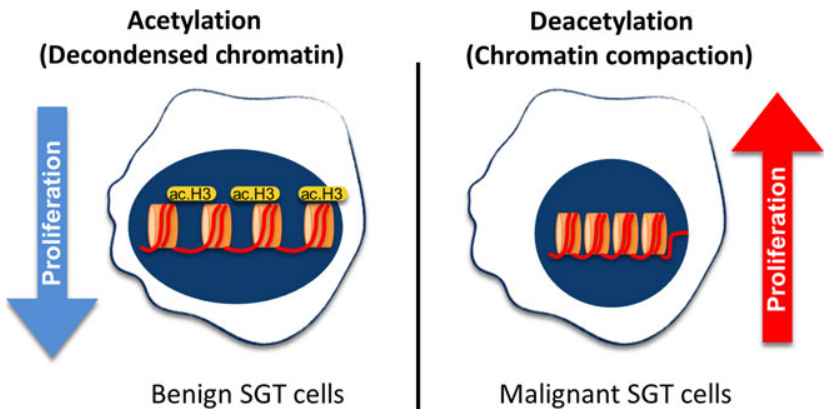

Figure 3 Schematic illustration of the proposed mechanism of histone acetylation and tumor proliferation. ac. H3, acetyl-histone H3 (lys9); SGT, Salivary Gland Tumor.

damage lesion (21). We believe that hypoacetylation of malignant tumors can operate to favor DNA damage perpetuation. Dnmt1 triggers histone deacetylase activity (22) and suppresses cell differentiation (23), leading to a stem cell phenotype. It is now well established that cancer stem cells are a major cause of tumor recurrence and metastasis (24) and are present in malignant SGTs $(25,26)$. Moreover, our group has demonstrated that acetyl-histone H3 (lys9) modifications are an important regulator of aggressiveness and resistance to cisplatin of head and neck squamous cell carcinoma via upregulation of the NFKB pathway (13). As for head and neck squamous cell carcinoma, platinum-based regimens are the most frequently employed for malignant SGTs (reviewed in (27)). No benefit, however, has been observed regarding survival in malignant SGT patients that respond to chemotherapy compared with those who had no response (28). The largest phase II trial that evaluated cisplatin in malignant SGTs revealed low response rates and short duration (29). The epigenetics modifications observed herein might indicate a promising target to increase cisplatin efficiency in malignant SGTs.

An interesting finding of the present study was the inverse correlation of $\mathrm{H} 3$ (lys9) acetylation and the proliferative activity of SGTs. We observed that tumor cells when more acetylated also demonstrate low proliferation rates. Overexpression of HDAC-1, which leads to tumor hypoacetylation, is associated with increased proliferative activity in pancreatic adenocarcinomas patients (7). The association of HDAC and increased proliferation was assessed in breast cancer, where it was demonstrated that HDAC9 is capable of regulating key genes such as cell cycle inhibitor $C D K N 1 A$ and the pro-apoptotic genes, BAX and DR4, in addition to SOX9, a gene associated with breast cancer cell proliferation and metastasis (30). Among the several effects of HDAC inhibitors, antiproliferative property is achieved by transcriptional upregulation of the cyclin-dependent kinase inhibitor p21, independently of p53 status (5).

The study of HDAC inhibitors is moving rapidly into a new stage of development. To date, three HDAC inhibitors have been approved by the US-FDA for the treatment of Tcell lymphoma. Vorinostat (SAHA), approved by the FDA in October 2006, also demonstrated promising results in several phase II trials in breast cancer (9). In fact, vorinostat produced better results when used as a sensitizer to reverse the resistance of hormone receptors inhibitors (9). Corroborating with this concept of HDAC inhibitors as adjuvant drugs that prevent chemoresistance, our group demonstrated that vorinostat inhibits cisplatin resistance in head and neck cancer cell lines (13). Recently, we showed that vorinostat administration has a greater impact in mucoepidermoid cancer stem cells compared with cisplatin alone and vorinostat plus cisplatin (11). These encouraging results 
show that in malignant SGTs, HDAC inhibitors might be used both as an adjuvant drug to overcome cisplatin resistance and as a single-agent therapy. Further studies are necessary to elucidate this important aspect.

Herein, we provided a comprehensive and novel vision regarding histone acetylation, a very significant epigenetic mechanism, in SGTs. Our results demonstrated that malignant SGTs are hypoacetylated, which leads to chromatin condensation. Moreover, we found that in salivary gland tumorigenesis, $\mathrm{H} 3$ acetylation impacts proliferation in an inversely proportional manner. Our results represent an initial step toward understanding epigenetic mechanisms involved in salivary gland tumorigenesis. We support that HDAC inhibition might represent a promising target to treat malignant SGTs.

\section{References}

1. Fonseca FP, Carvalho Mde V, De Almeida OP, et al. Clinicopathologic analysis of 493 cases of salivary gland tumors in a Southern Brazilian population. Oral Surg Oral Med Oral Pathol Oral Radiol 2012; 114: 230-9.

2. Terhaard CH, Lubsen H, Van Der Tweel I, et al. Salivary gland carcinoma: independent prognostic factors for locoregional control, distant metastases, and overall survival: results of the Dutch head and neck oncology cooperative group. Head Neck 2004; 26: 681-92; discussion 692-3.

3. Bell RB, Dierks EJ, Homer L, et al. Management and outcome of patients with malignant salivary gland tumors. J Oral Maxillofac Surg 2005; 63: 917-28.

4. Martins MD, Castilho RM. Histones: controlling tumor signaling circuitry. J Carcinog Mutagen 2013; 1: 1-12.

5. Glozak MA, Seto E. Histone deacetylases and cancer. Oncogene 2007; 26: 5420-32.

6. Fraga MF, Ballestar E, Villar-Garea A, et al. Loss of acetylation at Lys16 and trimethylation at Lys20 of histone H4 is a common hallmark of human cancer. Nat Genet 2005; 37: $391-400$.

7. Giaginis C, Damaskos C, Koutsounas I, et al. Histone deacetylase (HDAC)-1, -2, -4 and -6 expression in human pancreatic adenocarcinoma: associations with clinicopathological parameters, tumor proliferative capacity and patients' survival. BMC Gastroenterol 2015; 15: 148.

8. Chien W, Lee DH, Zheng Y, et al. Growth inhibition of pancreatic cancer cells by histone deacetylase inhibitor belinostat through suppression of multiple pathways including $\mathrm{HIF}, \mathrm{NFkB}$, and mTOR signaling in vitro and in vivo. Mol Carcinog 2014; 53: 722-35.

9. Munster PN, Thurn KT, Thomas S, et al. A phase II study of the histone deacetylase inhibitor vorinostat combined with tamoxifen for the treatment of patients with hormone therapyresistant breast cancer. Br J Cancer 2011; 104: 1828-35.

10. Zhang CY, Mao L, Li L, et al. Promoter methylation as a common mechanism for inactivating E-cadherin in human salivary gland adenoid cystic carcinoma. Cancer 2007; 110: 87-95.

11. Guimaraes DM, Almeida LO, Martins MD, et al. Sensitizing mucoepidermoid carcinomas to chemotherapy by targeted disruption of cancer stem cells. Oncotarget 2016; 7: 42447-60.

12. Giudice FS, Pinto DS Jr., Nor JE, et al. Inhibition of histone deacetylase impacts cancer stem cells and induces epithelialmesenchyme transition of head and neck cancer. PLOS ONE 2013; 8: e58672.

13. Almeida LO, Abrahao AC, Rosselli-Murai LK, et al. NFkap$\mathrm{paB}$ mediates cisplatin resistance through histone modifications in head and neck squamous cell carcinoma (HNSCC). FEBS Open Bio 2014; 4: 96-104.

14. Strahl BD. Allis, CD The language of covalent histone modifications. Nature 2000; 403: 41-5.

15. Paiva-Fonseca F, de Almeida OP, Ayroza-Rangel AL, et al. Tissue microarray construction for salivary gland tumors study. Med Oral Patol Oral Cir Bucal 2013; 18: e1-6.

16. Fonseca FP, de Andrade BA, Rangel AL, et al. Tissue microarray is a reliable method for immunohistochemical analysis of pleomorphic adenoma. Oral Surg Oral Med Oral Pathol Oral Radiol 2014; 117: 81-8.

17. Guzzo M, Locati LD, Prott FJ, et al. Major and minor salivary gland tumors. Crit Rev Oncol Hematol 2010; 74: 134-48.

18. Cesmebasi A, Gabriel A, Niku D, et al. Pediatric head and neck tumors: an intra-demographic analysis using the SEER* database. Med Sci Monit 2014; 20: 2536-42.

19. Yin LX, Ha PK. Genetic alterations in salivary gland cancers. Cancer 2016; 122: 1822-31.

20. Meshorer E, Misteli T. Chromatin in pluripotent embryonic stem cells and differentiation. Nat Rev Mol Cell Biol 2006; 7: 540-6.

21. Gong F, Miller KM. Mammalian DNA repair: HATs and HDACs make their mark through histone acetylation. Mutat Res 2013; 750: 23-30.

22. Fuks F, Burgers WA, Brehm A, et al. DNA methyltransferase Dnmt1 associates with histone deacetylase activity. Nat Genet 2000; 24: 88-91.

23. Sen GL, Reuter JA, Webster DE, et al. DNMT1 maintains progenitor function in self-renewing somatic tissue. Nature 2010; 463: 563-7.

24. Singh A, Settleman JEMT. Cancer stem cells and drug resistance: an emerging axis of evil in the war on cancer. Oncogene 2010; 29: 4741-51.

25. Adams A, Warner K, Nor JE. Salivary gland cancer stem cells. Oral Oncol 2013; 49: 845-53.

26. Adams A, Warner K, Pearson AT, et al. ALDH/CD44 identifies uniquely tumorigenic cancer stem cells in salivary gland mucoepidermoid carcinomas. Oncotarget 2015; 6: 26633-50.

27. Cerda T, Sun XS, Vignot S, et al. A rationale for chemoradiation (vs radiotherapy) in salivary gland cancers? On behalf of the REFCOR (French rare head and neck cancer network). Crit Rev Oncol Hematol 2014; 91: 142-58.

28. Carlson J, Licitra L, Locati L, et al. Salivary gland cancer: an update on present and emerging therapies. American Society of Clinical Oncology educational book/ASCO American Society of Clinical Oncology Meeting 2013; 257-63.

29. Licitra L, Marchini S, Spinazze S, et al. Cisplatin in advanced salivary gland carcinoma. A phase II study of 25 patients. Cancer 1991; 68: 1874-7.

30. Lapierre M, Linares A, Dalvai M, et al. Histone deacetylase 9 regulates breast cancer cell proliferation and the response to histone deacetylase inhibitors. Oncotarget 2016; 7: 19693-708.

\section{Acknowledgements}

This work was conducted during a visiting scholar period at the University of Michigan, sponsored by the Capes Foundation within the Ministry of Education, Brazil (grant n. BEX/99999.007990/2014-06). This grant was funded by the University of Michigan School of Dentistry faculty award. The funders had no role in study design, data collection, and analysis, decision to publish, or preparation of the manuscript.

\section{Conflict of interest}

All authors state they have no potential conflict of interests to declare. 